\title{
Introducing the Companions project: Intelligent, persistent, personalised interfaces to the internet.
}

\author{
David Benyon \\ Centre for Interaction Design \\ Napier University, Edinburgh \\ +441314552736 \\ d.benyon@napier.ac.uk
}

\author{
Oli Mival \\ Centre for Interaction Design \\ Napier University, Edinburgh \\ +441314552438 \\ o.mival@napier.ac.uk
}

\begin{abstract}
The Companions project is a 4 year, EU funded Framework Programme 6 project involving a consortium of 16 partners across 8 countries. Its aim is to develop a personalised conversational interface, one that knows and understands its owner, and can access resources on the Internet. It does this whilst nurturing an emotional, psychological and social involvement from its owner (user seems an inappropriate term in this context). In doing this it will change interactions to relationships. On a technical level it intends to push the state of the art in machine based natural language understanding, knowledge structures, speech recognition and text to speech.
\end{abstract}

\section{Keywords}

Agents, personalization, relationships.

\section{COMPANIONSHIP AND THE ELDERLY}

Companionship is a concept that is familiar to all, yet defies simple explanation. Psychology considers it a central need, yet balks at a concise definition of what constitutes a companion beyond "a relationship... with mutual caring and trust" [2], p467. What is clear, is the importance of companions to emotional well being. Indeed the loss of companions is considered a primary cause of depression among older people [6]. The erosion of social networks inevitably leads to the loss of companions and is often accompanied by an experience of emotional impoverishment, not infrequently experienced by the elderly as a pervasive depression "without a reason" [3]

With consideration of this natural decline in human companionship, the potential value of developing artificial companionship becomes distinctly apparent. On a simple level, older people have relationships with companions, be they pets, friends or care assistants. But what constitutes the difference between an interaction and a relationship? To form a relationship, the user needs to care about the interaction, to invest emotion in it. The artificial companions evoke the emotional investment through replicating recognizable real world behaviour. From this it may be suggested that the difference between a tool and a companion is a set of characteristics, a personality, which transforms an interaction

(C) David Benyon \& Oli Mival 2007 Published by the British Computer Society Volume 2 Proceedings of the 21st BCS HCI Group Conference HCI 2007, 3-7 September 2007, Lancaster University, UK Devina Ramduny-Ellis \& Dorothy Rachovides (Editors) into a relationship and evokes an emotional investment.

The medical benefits of pet ownership are well documented [3]. Pet ownership can lead to an enhanced emotional status and provides significant support in reducing emotional trauma following bereavement. Indeed not only emotional health but also physiological health is enhanced through contact with animals, particularly in the elderly. Furthermore, studies have shown that when animals enter the lives of older patients afflicted with Alzheimer's disease or arteriosclerosis, the patients will laugh and smile more, are more socially communicative and less hostile to their care workers [1,5]. However, some older people live in accommodation which does not allow pets or may suffer from psychological or physiological deterioration that make pet ownership problematic and potentially unsafe for the animal. In situations such as these, the use of artificial pets may be an alternative.

It is interesting to note that pets are at the non-specific purpose end of the companionship spectrum. A cat serves no other function than to be a cat. Yet as discussed above, by simply being a cat it can affect the health and well-being of its owner. People take delight in its activities, and it is purely from its behaviour that the benefits are derived. Cats cannot read email to you, struggle as a webcam and do not react to guidance from a computer. They are autonomous objects driven by their own goals. Kaplan suggests that this autonomy, this nonfunctionality is an important design consideration when developing artificial pets, he suggests they should "be designed as free 'not functional' creatures" [4].

It is the intention to use these insights to drive the interaction design elements of the major new FP6 EU project Companion.

\section{INTRODUCTION TO THE COMPANIONS PROJECT}

The Companions project, a 4 year, EU funded Framework Programme 6 project involving a consortium of 16 partners across 8 countries. The project's vision is that of a personalised conversational, multimodal interface to the Internet, one that knows its owner, is implemented on a range of platforms, indoor and nomadic, and based on integrated high-quality research in multimodal human-computer interfaces, intelligent agents, and human language technology [7]. This project is an ECA (Embodied Conversational Agent) which differs from the ECA state of the art by having large-scale speech and language capacity; it also differs significantly from the standard "big engineering" approach to this area, by offering relatively simple architectures with substantial tested performance, based on extensive application of powerful machine learning methods.

Companions will learn about their owners: their habits, their needs and their life memories. This will allow them to assist with carrying out specific Internet tasks, which will be facilitated by having complex models of their owners, by which 
we mean whole-life-memories, or coherent autobiographies, built from texts, conversations, images and videos. Some of this will already be in digital form, but some will be information gleaned from conversations with the Companion, information relatives and friends will want later, after the owner's death, but might never have been able to ask, such as "where did you and your husband first meet?"

The objectives of the proposal are to develop autonomous, persistent, affective and personal interfaces, or Companions, embedded in the Internet environment, with intelligent response in terms of speech and language, integrated with the manipulation of visual images and their content.

An early implementation of a Companion is PhotoPal [8]. PhotoPal allows people to view their photos and talk about them with their Companion. Photos are automatically tagged with the relevant dialogue allowing PhotoPal to build up a rich representation of the person's activities and relationships. This allows PhotoPal to sort, style and send photos and for people to reminisce with their Companion.

\section{Characteristics of Companions}

There are clearly many challenges for 'companion technology'. Bickmore and Picard [9]) argue that maintaining relationships involves managing expectations, attitudes and intentions. They emphasize that relationships are long-term built up over time through many interactions. Relationships are fundamentally social and emotional, persistent and personalized. Citing Kelley they say that relationships demonstrate interdependence between two parties - a change in one results in a change to the other. Relationships demonstrate unique patterns of interaction for a particular dyad, a sense of 'reliable alliance'. It is these characteristics of relationships as rich and extended forms of affective and social interaction that we are trying to tease apart. We do this by looking at the characteristics of companions in terms of utility, form, personality, emotion, social aspects and trust.

\subsection{Utility}

Utility is concerned with the allocation of function between the two participants in a relationship. Companions are needed to filter the large amount of information and conflicting views and ideas on the internet. They need to take the initiative and be proactive in starting some new activity, but only when appropriate. They may be conversational rather than task-focused.

\subsection{Form}

The form that a companion takes refers to all the issues of interaction such as dialogues, gestures, behviours and the other operational aspects of the interaction. It also refers to the representational aspects such as whether it is $2 \mathrm{D}$, graphical 3D or true $3 \mathrm{D}$, whether it has a humanoid, abstract or animal form, and the modalities that is uses. The many aesthetic issues are also considered under this heading. The form and the behaviours of the companion are likely to vary widely between different owners. The careful construction of a mixture of interface characteristics result in people enjoying the interaction and attributing intelligence and emotion to the Companion.

\subsection{Emotion}

Designing for pleasure and design for affect are key issues for companions [11]. Attractive things make people feel good which makes them more creative and more able (Norman, 2004). Relationships provide emotional support. Emotional integration and stability are key aspects of relationships [9]. Emotional aspects of the interaction come through meta relational communication, such as checking that everything is all right, use of humour and talking about the past and future. Another key aspect of an interaction if it is to become a relationship is empathy; empathy leads to emotional support and provides foundations for relationship-enhancing behaviours.

\subsection{Personality and Trust}

Personality is treated as a key aspect of the media equation by Reeves and Nass [12]. They undertook a number of studies that showed how assertive people prefer to interact with an assertive computer and submissive people prefer interacting with submissive devices. As soon as interaction moves from the utilitarian to the complexity of a relationship, people will want to interact with personalities that they like. Trust is "A positive belief about the perceived reliability of, dependability of, and confidence in a person, object or process" (Fogg, 2003). Trust is a key relationship that develops over time through small talk, getting acquainted talk and through acceptable 'continuity' behaviours. Routine behaviours and interactions contribute to developing a relationship where they are emphasizing commonalities and shared values.

\subsection{Social attitudes}

The social side of relationships such as group belonging, opportunities to nurture, autonomy support and social network support are all important. Relationships also play a key role in persuasion. The rather controversial idea of 'persuasive technologies' [10] is based on getting people to do things they would not otherwise do. In the context of companions, though, this is exactly what you would hope a companion would do providing it was ultimately for the good. A Health and Fitness companion, for example, should try to persuade its owner to run harder, or train more energetically.

\section{ACKNOWLEDGEMENTS}

This work is funded by the European Commission under contract IST 034434

\section{REFERENCES}

[1] T Garity, L Stallones, M Marx, M. \& P. Johnson, Pet Ownership and Attachment as Supportive Factors in the Health of the Elderly. Anthrozoos. Vol. 3, No.1 pps 35-44 (1989)

[2] H. Gleitman, Psychology. Oxford University Press (2000)

[3] K.L. Gory, \& K. Fitzpatrick, The effects of environmental contexts on elderly depression. Journal of Aging and Health, 4(4):459-479 (1992)

[4] F. Kaplan (2001) Free creatures: The role of uselessness in the design of artificial pets. In Proceedings of the CELETwente workshop on interacting agents 2001.

[5] O. Mival S. Cringean, and D. Benyon Personification Technologies: Developing Artificial Companions for Older People, ACM Press, 1--8. (2004)

[6] C. Sluzki, The extinction of the galaxy: Social networks in the elderly patient. New York: Family Process (2000)

[7] Y. Wilks, Artificial Companions as a new kind of interface to the future internet. Oxford Internet Institute, Research Report 13, October 2006

[8] www.napier.ac.uk/companions

[9] Bickmore T. and Picard R. (2005) Establishing and maintaining long-term human-computer relationships. ACM Transactions on Computer-Human Interaction (TOCHI), Volume 12 Issue 2

[10] Fogg, B. J. (2003) Persuasive Technologies. Morgan Kaufman

[11] Norman, D. (2004) Emotional Design Basic Books

[12] Reeves B. and Nass, C. (1996) The Media Equation CSLI Publications; Stanford, CA 